\title{
COVID-19-vaccination in patients receiving allergen immunotherapy (AIT) or biologics - EAACI recommendations
}

Marek Jutel ${ }^{1}$, María José Torres ${ }^{2}$, Oscar Palomares ${ }^{3}$, Cezmi Akdis ${ }^{4}$, Thomas Eiwegger ${ }^{5}$, Eva Untersmayr ${ }^{6}$, Domingo Barber Hernández ${ }^{7}$, Magdalena Zemelka-Wiacek ${ }^{8}$, Anna Kosowska $^{1}$, Elizabeth Palmer ${ }^{9}$, Stefan Vieths ${ }^{10}$, Vera Mahler ${ }^{11}$, Giorgio Walter Canonica ${ }^{12}$, Kari Nadeau ${ }^{13}$, Mohamed Shamji ${ }^{9}$, Ioana Agache ${ }^{14}$, Mubeccel Akdis ${ }^{15}$, Musa Khaitov ${ }^{16}$, Alberto Alvarez-Perea ${ }^{17}$, Montserrat Alvaro ${ }^{18}$, Marina Atanasković-Marković ${ }^{19}$, Vibeke Backer $^{20}$, Annick BARBAUD ${ }^{21}$, Sevim Bavbek ${ }^{22}$, Frédéric De Blay ${ }^{23}$, Matteo Bonini ${ }^{24}$, Sergio Bonini ${ }^{25}$, Job van Boven ${ }^{26}$, Knut Brockow ${ }^{27}$, Mario Cazzola ${ }^{28}$, Alexia Chatzipetrou $^{29}$, T. Chivato ${ }^{30}$, A. Cianferoni ${ }^{31}$, Jonathan Corren ${ }^{32}$, Jean-Christoph Caubet $^{33}$, Audrey DunnGalvin ${ }^{34}$, Motohiro Ebisawa ${ }^{35}$, Davide Firinu ${ }^{36}$, Radoslaw Gawlik $^{37}$, Asli Gelincik ${ }^{38}$, Stefano Del Giacco ${ }^{36}$, Charlotte Mortz ${ }^{39}$, H.J. Hoffmann ${ }^{40}$, Karin Hoffmann-Sommergruber ${ }^{41}$, Ludger Klimek ${ }^{42}$, Antti Lauerma ${ }^{43}$, Luis Perez de Llano ${ }^{44}$, Andrea Matucci ${ }^{45}$, Rosan Meyer ${ }^{46}$, André Moreira ${ }^{47}$, Hideaki Morita ${ }^{48}$, Sarita Patil ${ }^{49}$, Oliver Pfaar ${ }^{50}$, Florin-Dan Popescu ${ }^{51}$, VICTORIA DEL POZO ${ }^{52}$, Oliver Price ${ }^{53}$, Ronald van Ree ${ }^{54}$, Montserrat Fernandez-Rivas ${ }^{55}$, Barbara Rogala ${ }^{37}$, Antonino Romano ${ }^{56}$, Alexandra Santos ${ }^{57}$, Anna Sediva ${ }^{58}$, Isabel Skypala ${ }^{59}$, Sylwia Smolinska ${ }^{60}$, Milena Sokolowska $^{61}$, Gunter Sturm ${ }^{62}$, Alessandra Vultaggio ${ }^{63}$, Jolanta Walusiak-Skorupa ${ }^{64}$, and Margitta Worm ${ }^{65}$

${ }^{1}$ Wroclaw Medical University

${ }^{2}$ Universidad de Málaga Facultad de Medicina

${ }^{3}$ Complutense University of Madrid

${ }^{4}$ University of Zurich

${ }^{5}$ The Hospital for Sick Children

${ }^{6}$ Center of Physiology, Pathophysiology and Immunology

${ }^{7}$ Universidad San Pablo CEU

${ }^{8}$ University of Wroclaw

${ }^{9}$ Imperial College London

${ }^{10}$ Paul-Ehrlich-Institut,

${ }^{11}$ Paul-Ehrlich-Institut

${ }^{12}$ Humanitas University Head Personalized Medicine Asthma \& Allergy Clinic-Humanitas Research Hospital-IRCCS, Milano, Italy

${ }^{13}$ Stanford University

${ }^{14}$ Transylvania University of Brasov

${ }^{15}$ University of Zürich

${ }^{16} \mathrm{NRC}$ Institute of Immunology

${ }^{17}$ Hospital General Universitario Gregorio Marañón

${ }^{18}$ Hospital Sant Joan de Deu

${ }^{19}$ University of Belgrade, Facuty of Medicine 
${ }^{20}$ Bispebjerg University Hospital

${ }^{21}$ Sorbonne Universités, UPMC Univ Paris 06

${ }^{22}$ Ankara University Faculty of Medicine

${ }^{23}$ University Hospital Strasbourg

${ }^{24}$ Imperial College London Faculty of Medicine

${ }^{25}$ National Research Council

${ }^{26}$ Groningen Research Institute for Asthma and COPD (GRIAC), University Medical Center Groningen, University of Groningen, Groningen, The Netherlands

${ }^{27}$ Technical University of Munich

${ }^{28}$ University of Rome Tor Vergata

${ }^{29}$ National University of Athens, Medical School

${ }^{30}$ Univ CEU San Pablo

31 the childrens' Hospital of Philadelphia

${ }^{32}$ University of California, Los Angeles

${ }^{33}$ Univ Hosp Geneva

${ }^{34}$ University College Cork (UCC)

${ }^{35}$ Sagamihara National Hospital

${ }^{36}$ University of Cagliari

${ }^{37}$ Medical University of Silesia

${ }^{38}$ Istanbul University

${ }^{39}$ Odense Universityhospital

${ }^{40}$ European Academy of Allergy and Clinical Immunology; Department of Clinical Medicine, Aarhus University

${ }^{41}$ Medical University Vienna

${ }^{42}$ Center for Rhinology and Allergology Wiesbaden

${ }^{43}$ University of Helsinki

${ }^{44}$ Hospital Universitario Lucus Augusti

${ }^{45}$ Azienda Ospedaliero-Universitaria Careggi

${ }^{46}$ Imperial College

${ }^{47}$ Faculty of Medicine

${ }^{48}$ National Research Institute for Child Health and Development

${ }^{49}$ Massachusetts General Hospital and Harvard Medical School

${ }^{50}$ University Hospital Marburg, Philipps-Universität Marburg, Marburg, Germany.

${ }^{51}$ Universitatea de Medicina si Farmacie Carol Davila

${ }^{52}$ Fundación Jiménez Díaz

${ }^{53}$ University of Leeds

${ }^{54}$ Academic Medical Center

${ }^{55}$ Hospital Clinico San Carlos

${ }^{56}$ Allergy Unit Complesso Integrato Columbus, Rome and IRCCS Oasi Maria S.S., Troina, Italy

${ }^{57}$ King's College London Faculty of Life Sciences and Medicine

${ }^{58}$ Motol University Hospital Department of Cardiology 2nd Faculty of Medicine

${ }^{59}$ Royal Brompton \& Harefield

${ }^{60}$ Uniwersytet Medyczny im Piastow Slaskich we Wroclawiu 


\author{
${ }^{61}$ Swiss Institute of Allergy and Asthma Research, University of Zurich \\ ${ }^{62}$ Medizinische Universitat Graz \\ ${ }^{63}$ University Hospital Careggi \\ ${ }^{64}$ Nofer Institute of Occupational Medicine \\ ${ }^{65}$ Charité - Universitätsmedizin Berlin
}

June 24, 2021

\begin{abstract}
Immune modulation is a key therapeutic tool for allergic diseases and asthma. It can be achieved in an antigen-specific way via allergen immunotherapy (AIT) or in endotype-driven approach using biologicals that target the major pathways of the type 2 (T2) immune response: IgE, IL-5 and IL-4/IL-13. COVID-19 vaccine provides an excellent opportunity to tackle the global pandemics and is currently being applied in an accelerated rhythm worldwide. It works as well through immune modulation. Thus, as there is an obvious interference between these treatment modalities recommendations on how they should be applied in sequence are expected. The European Academy of Allergy and Clinical Immunology (EAACI) gathered an outstanding expert panel under its Research and Outreach Committee (ROC). This expert panel was called to evaluate the evidence and formulate recommendation on the administration of COVID-19 vaccine in patients with allergic diseases and asthma receiving AIT or biologicals. The panel also formulated recommendations for COVID-19 vaccine in association with biologicals targeting the type 1 or type 3 immune response. In formulating recommendations, the panel evaluated the mechanisms of COVID-19 infection, of COVID-19 vaccine, of AIT and of biologicals and considered the data published for other anti-infectious vaccines administered concurrently with AIT or biologicals.
\end{abstract}

\title{
Hosted file
}

COVID-19 vaccination_EAACI_recom_final-docx.docx available at https://authorea.com/ users/327584/articles/527558-covid-19-vaccination-in-patients-receiving-allergen-

immunotherapy-ait-or-biologics-eaaci-recommendations 